\title{
Measurement of thermal diffusivity by a modification of the Angstroem's method using thermally short specimens
}

by Alberto Muscio*

\author{
${ }^{*}$ DIMeC - Dipartimento di Ingegneria Meccanica e Civile \\ Università di Modena e Reggio Emilia \\ Via Vignolese 905/B, 41100 Modena, Italy \\ Phone+39-059-2056194, Fax+39-059-2056126, E-mail: alberto.muscio@unimore.it
}

\section{Keywords}

Thermal diffusivity, Angstroem's method, infrared thermography, mathematical model, finite specimen

\section{Abstract}

A modification of the Angstroem's method for measurement of thermal diffusivity is presented. The Angstroem's method relies upon the steady-periodic propagation of temperature waves along the specimen. The diffusivity is recovered from combination of phase shift and amplitude decay of the waves, measured by infrared thermography, under the hypothesis of virtually semi-infinite specimen, that is neglecting the reflection of temperature waves at the end of the specimen.

In this work, a condition is exploited in which the specimen is not considered semi-infinite. Testing of a large set of materials is made possible, as well as recovering the diffusivity value from either amplitude decay or phase shift, independently. A procedure to estimate the diffusivity is developed from the mathematical model of the 'thermally short' specimen and it is subsequently validated by numerical simulation.

\section{Introduction}

Thermal diffusivity is a property difficult to measure, especially for materials such as pure metals, crystalline oxides, semiconductors or layered composites. These materials have high value of the diffusivity and are often available as plane thin specimens. Moreover, they are frequently anisotropic, with diffusivity through the thickness different from that in parallel to the plane of the specimen. The standard 'flash method' allows measuring the diffusivity through the thickness, but not in the specimen plane. It also requires a careful preparation of the experiment. Other techniques have been studied, but so far an alternative standard has not been established.

A good candidate for a new standard test method is the technique proposed by Angstroem in 1861 [1]. It relies upon the steady-periodic propagation of temperature waves. The diffusivity is recovered from phase shift and amplitude decay of the waves along the specimen. More specifically, Angstroem employed a long bar of small cross-section. One end of the bar was subjected to a periodic change of temperature, being alternately heated by a current of steam and cooled by a current of cold water for equal time intervals. After a few cycles the temperature field settles down to a steady-periodic state, which was monitored by Angstroem at selected points along the bar.

The Angstroem's method was rediscovered and modified in several manners since the beginning of the past century, as modern laboratory instrumentation made more and more effective the generation and monitoring of the temperature waves [2-12]. A more accurate assessment of phase shift and amplitude decay of the waves was recently allowed by computerized data-acquisition and data-processing systems. A significant advancement was also given by the introduction of non-contact temperature detectors and thermographic instruments.

Contact heaters, electron bombardment, light beams being cyclically modulated or periodically shielded by a moving mask, and chopped laser beams were largely employed to generate the thermal waves [2-8]. Sources with a positive net heat input, however, produce a large stationary gradient of temperature along the specimen. This may influence heat transfer between specimen and surrounding medium, causing significant discrepancy from the theoretical models. A great number of cycles is also needed to achieve steady-periodic conditions. Above all, it is difficult to produce a sinusoidal temperature oscillation and the analysis of data is made difficult. Therefore, contact sources based on the Peltier effect were also employed [9-12]. These allow one to alternate heating and cooling stages, thus obtaining a null net heat input and temperature cycles about the ambient value. The heat rate of Peltier heat pumps is also proportional to the applied current, a peculiarity that has recently been exploited to achieve a perfectly harmonic temperature cycle [13-16]. As a result, it is possible to estimate the diffusivity through a relatively simple fitting of surface temperature measurements. These can be easily acquired along the surface of the specimen, with high frequency and accuracy, by means of modern thermographic systems.

The Angstroem's method and its modifications are based on the assumption of virtually semi-infinite specimen, that is on neglecting the reflection of temperature waves at the end of the specimen opposite to the thermal source. This makes simple the mathematical model through which the diffusivity is estimated, but it requires relatively long specimens and long time cycles to achieve a satisfactory signal-to-noise ratio. Conductive 
materials available in small samples are often difficult to analyze. On the other hand, exploiting a condition in which the specimen is not considered semi-infinite permits to test a larger set of materials and, above all, to recover the diffusivity value from either amplitude decay or phase shift of the temperature waves, independently. This is often impossible when a condition of 'thermally semi-infinite' specimen is achieved, as the effects of heat transfer between specimen and test ambient must be filtered by combining amplitude decay and phase shift data. Moreover, when surface temperature data are acquired by infrared thermography, an uneven emissivity of the specimen surface may affect significantly the measurement of amplitude decay, but not that of phase shift.

In this work the mathematical model for the 'thermally short' specimen condition is presented. It is obtained by solution of the Fourier's equation of heat transfer. From the model, a procedure to estimate the thermal diffusivity is developed and subsequently validated by numerical simulation.

\section{Mathematical formulation for the 'thermally semi-infinite' specimen}

The Angstroem's method is generally applied to a specimen with small cross-section and length great enough to be considered virtually semi-infinite. A temperature cycle is imposed at one end of the specimen, which exchanges heat with the test environment by convection and radiation through its surfaces exposed to air. Assuming a uniform temperature in the cross section, the temperature distribution along the specimen is governed by the well-known equation of heat diffusion in the following form:

$$
\frac{\partial T(x, t)}{\partial t}=\alpha \frac{\partial^{2} T(x, t)}{\partial x^{2}}-\frac{h P}{\rho c S}\left[T(x, t)-T_{a}\right]
$$

$T[\mathrm{~K}]$ is the local temperature of the specimen, $T_{a}[\mathrm{~K}]$ is the ambient temperature, $t[\mathrm{~s}]$ is time, $x[\mathrm{~m}]$ is the coordinate along the direction of wave propagation, $P[\mathrm{~m}]$ and $S\left[\mathrm{~m}^{2}\right]$ are the perimeter and the area of the crosssection, $h\left[\mathrm{~W} /\left(\mathrm{m}^{2} \mathrm{~K}\right)\right]$ is the coefficient of surface heat transfer at the specimen surface, $\alpha\left[\mathrm{m}^{2} / \mathrm{s}\right], \rho\left[\mathrm{kg} / \mathrm{m}^{3}\right]$, and c $[\mathrm{J} /(\mathrm{kg} \mathrm{K})]$ are, respectively, the thermal diffusivity, the density, and the specific heat of the material.

The boundary condition at the end cyclically heated and cooled can be expressed by a Fourier's series. In connection with that, a steady-periodic solution of Eq. (1), independent of the initial condition, always exists [17], but it is relatively complex and unpractical to estimate the diffusivity. The solution is greatly simplified, however, if the boundary condition at $x=0$ is that of a perfectly harmonic temperature cycle about a given value $T_{0}[\mathrm{~K}]$ :

$$
T(x=0, t)=T_{0}+\Delta T_{0} \sin \left(2 \pi \frac{t}{t_{0}}+\varphi_{0}\right)
$$

where $\Delta T_{0}[\mathrm{~K}], t_{0}[\mathrm{~s}]$, and $\varphi_{0}[\mathrm{rad}]$ are, respectively, amplitude, time-cycle, and initial phase.

At the opposite end, the temperature is not affected by the alterations at $x=0$ if the specimen is long enough (i.e., virtually semi-infinite). The boundary condition thus is:

$$
T(x \rightarrow \infty, t)=T_{a}
$$

The steady-periodic temperature field yielded along the specimen is [1]:

$$
T(x, t)=T_{a}+\left(T_{0}-T_{a}\right) \exp \left(-\sqrt{\frac{h P}{\lambda S}} x\right)+\Delta T_{0} \exp \left(-\gamma \frac{x}{L}\right) \cos \left(2 \pi \frac{t}{t_{0}}-\frac{1}{\gamma} \frac{x}{L}+\varphi_{0}\right)
$$

where $\lambda[\mathrm{W} /(\mathrm{m} \mathrm{K})]$ is the thermal conductivity of the material.

Equation (4) describes a harmonic thermal wave, exponentially decaying while it travels along the specimen (figure 1). A continuous (dc) term is also superposed to the steady-periodic (ac) thermal field, consisting of an exponential decay of the mean temperature from $T_{0}$ to $T_{a}$.

Amplitude decay and phase shift are proportional to the so-called thermal diffusion length, $L[\mathrm{~m}]$. This is a characteristic parameter that depends on the thermal diffusivity of the specimen, $\alpha$, and the time-cycle $t_{0}$ :

$$
L=\sqrt{\frac{\alpha_{s} t_{0}}{\pi}}
$$

The effects of heat exchange with the test ambient are included in the dimensionless parameters $\gamma(\geq 1)$, which affects either the phase or the amplitude of the thermal waves: 


$$
\gamma=\sqrt{\frac{t_{0}}{t_{h}}+\sqrt{1+\left(\frac{t_{0}}{t_{h}}\right)^{2}}} \Leftrightarrow \frac{1}{\gamma}=\sqrt{-\frac{t_{0}}{t_{h}}+\sqrt{1+\left(\frac{t_{0}}{t_{h}}\right)^{2}}}
$$

The relaxation time $t_{h}[\mathrm{~s}]$ in Eq. (6) is defined as follows:

$$
t_{h}=2 \pi \frac{\rho c S}{h P}
$$

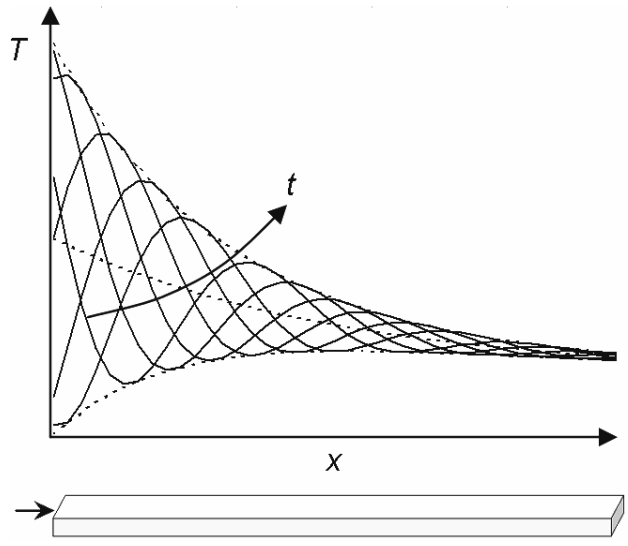

Fig. 1. Propagation of a harmonic temperature wave with exponential damping.

The diffusivity can be estimated from the thermal diffusion length, $L$. This is in turn calculated from the slope of the normalized wave amplitude, $\Delta \vartheta_{x}=\Delta T(x) / \Delta T_{0}$, or from the slope of the phase shift, $\varphi_{x}$. Two apparent values of the diffusivity, $\alpha_{\Delta}$ and $\alpha_{\varphi}$, are eventually found:

$$
\begin{aligned}
& \frac{\gamma}{L}=-\frac{\partial\left(\ln \Delta \vartheta_{X}\right)}{\partial x} \Leftrightarrow \alpha_{\Delta}=\frac{1}{\gamma^{2}} \alpha=\frac{\pi / t_{0}}{\left[d\left(\ln \Delta \vartheta_{X}\right) / d x\right]^{2}} \\
& \frac{1}{\gamma L}=\frac{d \varphi_{X}}{d x} \Leftrightarrow \alpha_{\varphi}=\gamma^{2} \alpha=\frac{\pi / t_{0}}{\left(d \varphi_{x} / d x\right)^{2}}
\end{aligned}
$$

The apparent values are related to the actual one by two unknown factors, $1 / \gamma^{2}$ and $\gamma^{2}$, both ones dependent of surface heat transfer. As shown in figure 2, the actual diffusivity is respectively overestimated by $\alpha_{\Delta}$ $\left(1 / \gamma^{2} \leq 1\right)$ or underestimated by $\alpha_{\varphi}\left(\gamma^{2} \geq 1\right)$ :

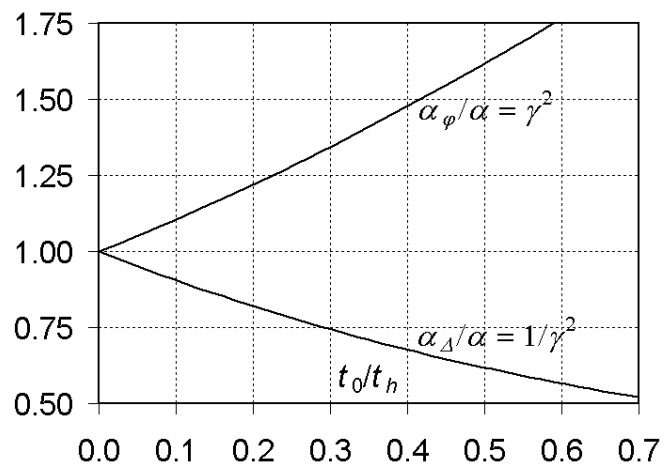

Fig. 2. Impact of heat exchange with the test ambient on the diffusivity estimate.

The true diffusivity can nevertheless be calculated by combining $\alpha_{\Delta}$ and $\alpha_{\varphi}$ :

$$
\alpha=\sqrt{\alpha_{\Delta} \alpha_{\varphi}}
$$


Heat exchange with the test ambient can be made negligible, and $\gamma$ about equal to unity, by choosing a time cycle $t_{0}$ much smaller than $t_{h}$ (figure 2). This yields values of apparent diffusivity almost coincident with the actual one and allows two independent estimates. In practice, however, the effects of surface heat transfer impose relatively large values of the ratio $t_{0} / t_{h}$ and Eq. (10) must generally be used.

Equation (4) can be simplified further if the temperature oscillation imposed at $x=0$ is about the ambient temperature $T_{a}$ (i.e. $T_{0} \equiv T_{a}$ ) and, consequently, the dc-term is made null:

$$
T(x, t)=T_{a}+\Delta T_{0} \exp \left(-\gamma \frac{x}{L}\right) \cos \left(2 \pi \frac{t}{t_{0}}-\frac{1}{\gamma} \frac{x}{L}+\varphi_{0}\right)
$$

Eliminating the dc-term may allow a faster attainment of steady-periodic conditions. It makes also easier the processing of surface-temperature data to extract the apparent diffusivities. On the other hand, the decay of mean temperature given by the dc-term can provide a confirmation of the diffusivity estimate as it depends on $\alpha$ and $t_{h}$ :

$$
\sqrt{\frac{h P}{\lambda S}} \equiv \sqrt{\frac{2 \pi}{\alpha t_{h}}} \Leftrightarrow \alpha=\frac{2 \pi}{t_{h}}\left(\frac{\lambda S}{h P}\right) \equiv \frac{\alpha_{\varphi}-\alpha_{\Delta}}{2 \sqrt{\alpha_{\Delta} \alpha_{\varphi}}}\left(\frac{\lambda S}{h P}\right)
$$

\section{Mathematical formulation for the 'thermally short' specimen}

If the thermal waves reach the end of the specimen opposed to the source before being completely damped, they are reflected back. As a result, the temperature distribution may by considerably different from that described by Eq. (4) or Eq. (11). The effects of reflection can be minimized using specimens with proper length and/or waves with short time-cycle. The amplitude of direct waves is damped to one hundredth of the initial value $\Delta T_{0}$ at a distance from the source equal to about 4.5 times the thermal diffusion length $L$, which is in turn proportional to the square root of the time cycle $t_{0}$, or to one thousandth of $\Delta T_{0}$ at a distance equal to about 7 times $L$. Specimens with proper size are often unavailable, however, or long time-cycles are needed to obtain an adequate signal to noise ratio.

When it is not possible to achieve the condition of thermally semi-infinite specimen, the following boundary condition occurs at the specimen end opposite to the source, located at a distance $X[\mathrm{~m}]$ from the source itself:

$$
-\lambda \frac{\partial T(x=X, t)}{\partial x}=h^{\prime}\left[T(x=X, t)-T_{a}\right]
$$

The heat transfer coefficient at the side surfaces, $h\left[\mathrm{~W} /\left(\mathrm{m}^{2} \mathrm{~K}\right)\right]$, may differ significantly from that at the end surface, $h^{\prime}$, due to a different surface finish or a different air velocity, respectively affecting the radiative component and the convective component of the coefficient. In the following it is assumed that $h \neq h^{\prime}$.

Solving with respect to $T(x)$ the system of Eqs. (1)-(2) and eq. (13), one obtains the distribution and the time-evolution pattern of temperature in the specimen. This can be achieved by making dimensionless the equation system.

$$
\begin{aligned}
& \frac{\partial \vartheta(\xi, \tau)}{\partial \tau}=\pi \frac{\partial^{2} \vartheta(\xi, \tau)}{\partial \xi^{2}}-\frac{2 \pi}{\tau_{h}} \vartheta(\xi, \tau) \\
& \vartheta(\xi=0, \tau)=\cos (2 \pi \tau) \\
& \frac{\partial \vartheta(\xi=\Xi, \tau)}{\partial \xi}=-B i_{L} \vartheta(\xi=\Xi, \tau)
\end{aligned}
$$

The following dimensionless terms were used:

$$
\vartheta=\frac{T-T_{a}}{\Delta T_{0}} \quad \tau=\frac{t}{t_{0}} \quad \tau_{h}=\frac{t_{h}}{t_{0}} \quad \xi=\frac{x}{L} \quad \Xi=\frac{X}{L} \quad B i_{L}=\frac{h^{\prime} L}{\lambda}
$$

$\vartheta$ represents the local temperature difference between specimen and ambient normalized by the initial amplitude $\Delta T_{0}$. The dimensionless time $\tau$ and external relaxation time $\tau_{h}$ are the ratios of the corresponding dimensional values by the time cycle $t_{0}$. The dimensionless space coordinate $\xi$ and specimen length $\Xi$ are the rations of the corresponding dimensional values by the thermal diffusion length $L$. Finally, $B i_{L}$ is the Biot number and it takes into account heat exchange with the test ambient at the end surface of the specimen.

The following auxiliary system of dimensionless equations can be associated to the main one: 


$$
\begin{aligned}
& \frac{\partial \hat{\vartheta}(\xi, \tau)}{\partial \tau}=\pi \frac{\partial^{2} \hat{\vartheta}(\xi, \tau)}{\partial \xi^{2}}-\frac{2 \pi}{\tau_{h}} \hat{\vartheta}(\xi, \tau) \\
& \hat{\vartheta}(\xi=0, \tau)=\sin (2 \pi \tau) \\
& \frac{\partial \hat{\vartheta}(\xi=\Xi, \tau)}{\partial \xi}=-B i i_{L} \hat{\vartheta}(\xi=\Xi, \tau)
\end{aligned}
$$

Adding to each equation of the main system the corresponding equation of the auxiliary system multiplied by the imaginary unit $i=\sqrt{-1}$, the following system of complex equations is obtained:

$$
\begin{aligned}
& \frac{\partial \tilde{\vartheta}(\xi, \tau)}{\partial \tau}=\pi \frac{\partial^{2} \tilde{\vartheta}(\xi, \tau)}{\partial \xi^{2}}-\frac{2 \pi}{\tau_{h}} \tilde{\vartheta}(\xi, \tau) \\
& \tilde{\vartheta}(\xi=0, \tau)=\exp (i 2 \pi \tau) \\
& \frac{\partial \tilde{\vartheta}(\xi=\Xi, \tau)}{\partial \xi}=-B i_{L} \tilde{\vartheta}(\xi=\Xi, \tau)
\end{aligned}
$$

where

$$
\begin{aligned}
& \tilde{\vartheta}(\xi, \tau)=\vartheta(\xi, \tau)+i \hat{\vartheta}(\xi, \tau) \\
& \exp (i 2 \pi \tau)=\cos (2 \pi \tau)+i \sin (2 \pi \tau)
\end{aligned}
$$

The steady-periodic solution of the main problem, valid for $\tau \rightarrow \infty$, is the real part of the solution for the complex equation system, obtained by separation of variables and having the following general form:

$$
\tilde{\vartheta}(\xi, \tau)=\psi(\xi) \exp (i 2 \pi \tau)
$$

Introducing Eq. (31) into Eqs. (26)-(28) and simplifying, one has:

$$
\begin{aligned}
& \frac{d^{2} \psi(\xi)}{d \xi^{2}}=2\left(\mathrm{i}+\frac{1}{\tau_{h}}\right) \psi(\xi) \\
& \psi(\xi=0)=1 \\
& \frac{d \psi(\xi=\Xi)}{d \xi}=-B i_{L} \psi(\xi=\Xi)
\end{aligned}
$$

Solving with respect to $\psi(\xi)$, one obtains:

$$
\psi(\xi)=\frac{\left(\gamma+i \frac{1}{\gamma}\right) \cosh \left[\left(\gamma+i \frac{1}{\gamma}\right)(\Xi-\xi)\right]+B i_{L} \sinh \left[\left(\gamma+i \frac{1}{\gamma}\right)(\Xi-\xi)\right]}{\left(\gamma+i \frac{1}{\gamma}\right) \cosh \left[\left(\gamma+i \frac{1}{\gamma}\right) \Xi\right]+B i_{L} \sinh \left[\left(\gamma+i \frac{1}{\gamma}\right) \Xi\right]}
$$

Equation (35) can then be introduced into the general solution given in Eq. (31):

$$
\tilde{\vartheta}(\xi, \tau)=\frac{\left(\gamma+i \frac{1}{\gamma}\right) \cosh \left[\left(\gamma+i \frac{1}{\gamma}\right)(\Xi-\xi)\right]+B i_{L} \sinh \left[\left(\gamma+i \frac{1}{\gamma}\right)(\Xi-\xi)\right]}{\left(\gamma+i \frac{1}{\gamma}\right) \cosh \left[\left(\gamma+i \frac{1}{\gamma}\right) \Xi\right]+B i_{L} \sinh \left[\left(\gamma+i \frac{1}{\gamma}\right) \Xi\right]} \exp (i 2 \pi \tau)
$$

The solution to the main problem is the real part of Eq. (36). After some mathematical manipulation and the reintroduction of the initial phase $\varphi_{0}$, that solution can be expressed in the form:

$$
\vartheta(\xi, \tau)=\Delta \vartheta(\xi) \cos \left[2 \pi \tau+\varphi(\xi)+\varphi_{0}\right]
$$

where non-dimensional amplitude decay $\Delta \vartheta(\xi)$ and phase shift $\varphi(\xi)$ are given in Eqs. (38)-(39): 


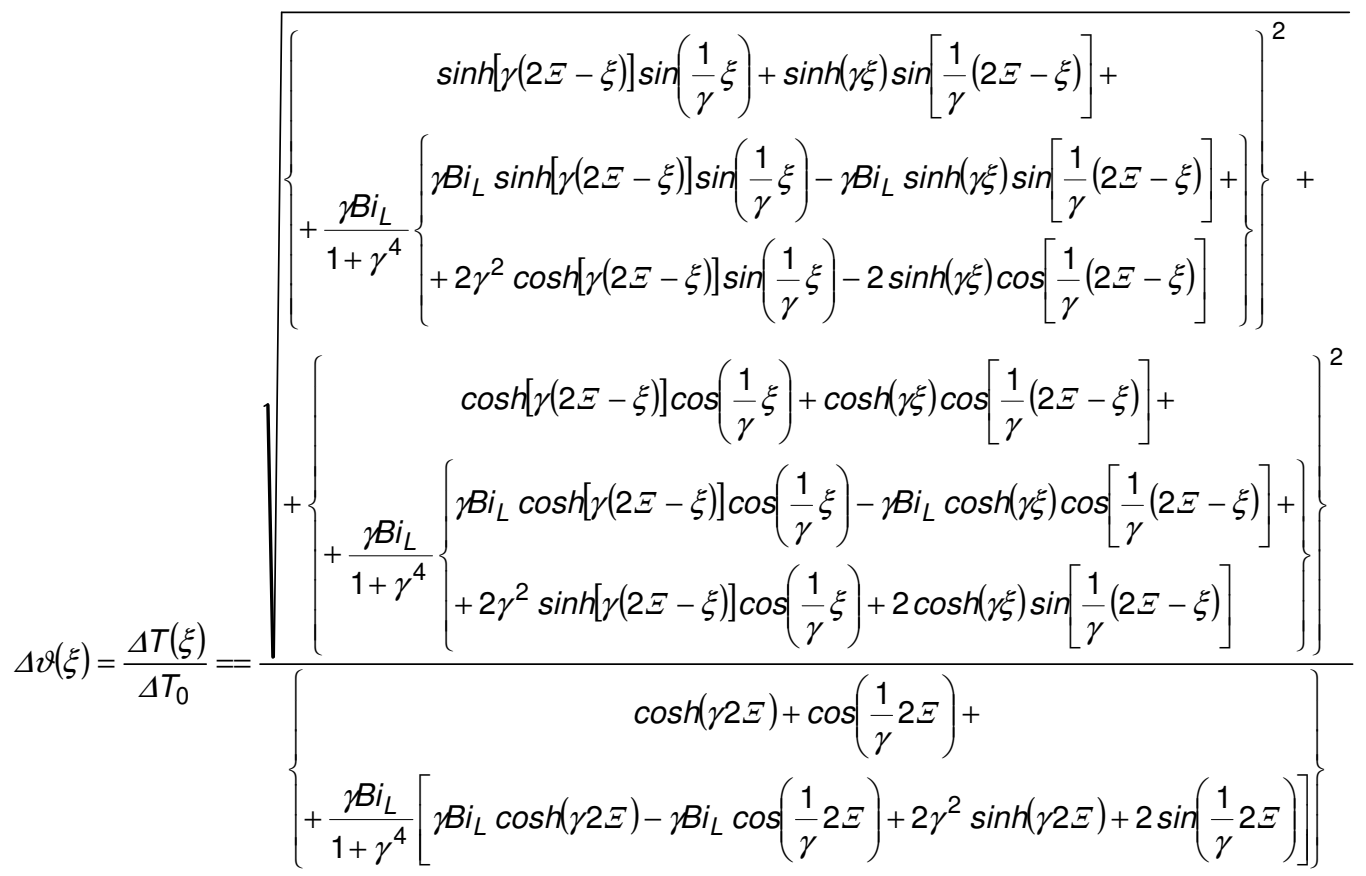

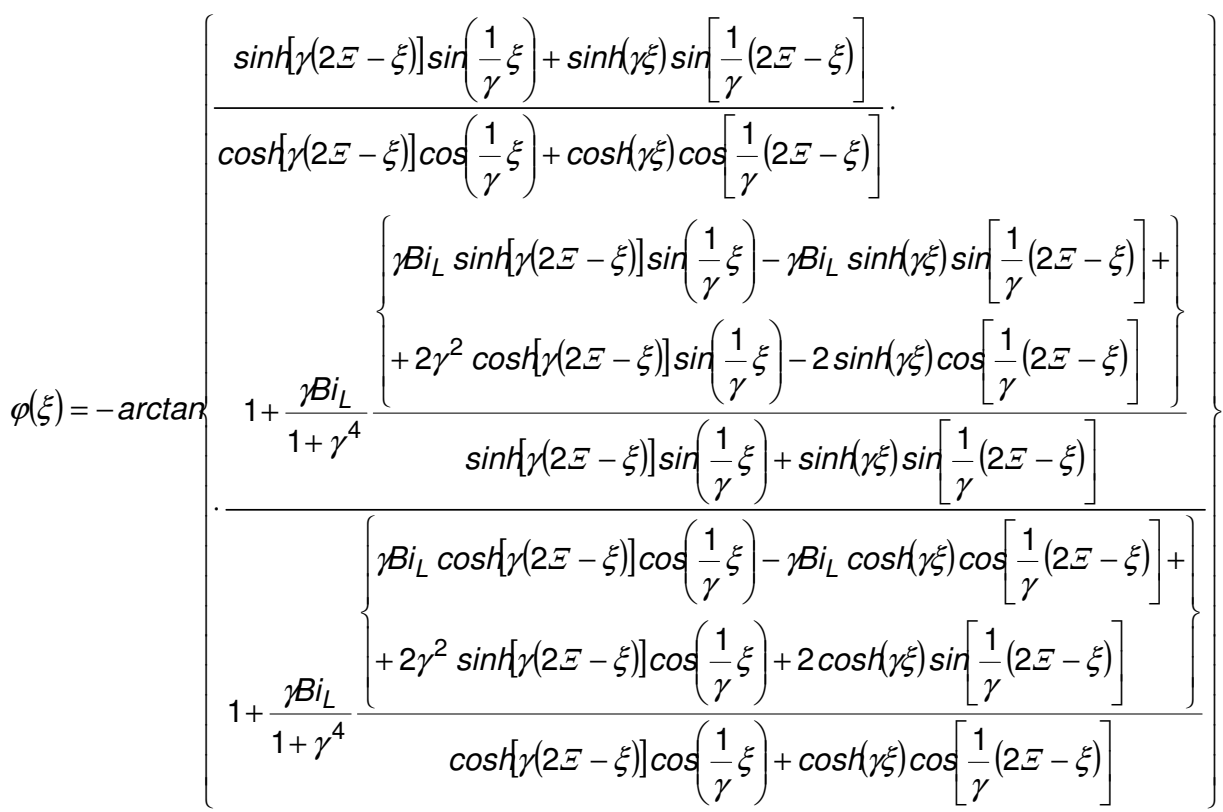

Equations (38)-(39) can be simplified considerably if heat exchange with the test ambient can be neglected (i.e. $\left.\gamma \rightarrow 1, B i_{L} \rightarrow 0\right)$ :

$$
\begin{aligned}
& \Delta \vartheta(\xi)=\frac{\sqrt{[\sinh (2 \Xi-\xi) \sin (\xi)+\sinh (\xi) \sin (2 \Xi-\xi)]^{2}+[\cosh (2 \Xi-\xi) \cos (\xi)+\cosh (\xi) \cos (2 \Xi-\xi)]^{2}}}{\cosh (2 \Xi)+\cos (2 \Xi)} \\
& \varphi(\xi)=-\arctan \left[\frac{\sinh (2 \Xi-\xi) \sin (\xi)+\sinh (\xi) \sin (2 \Xi-\xi)}{\cosh (2 \Xi-\xi) \cos (\xi)+\cosh (\xi) \cos (2 \Xi-\xi)}\right]
\end{aligned}
$$

The temperature oscillation described by Eq. (37) is everywhere perfectly harmonic. Therefore, the origin of the reference system $(\xi=0)$ can be placed at any point along the specimen. This allows excluding the portion of the specimen closest to the source, where thermographic measurements may be disturbed by reflection of infrared radiation from the source itself or the wave front of the temperature oscillations may be not yet perfectly flat and perpendicular to the specimen length [13-16]. The initial amplitude $\Delta T_{0}$ and phase $\varphi_{0}$ must be evaluated at the chosen origin. 
The effects of wave reflection at the end of a thermally short specimen can be highlighted through the ratio of $\varphi(\xi)$ as described by Eq. (39) or Eq. (41) and the phase in a thermally semi-infinite specimen $(\xi \rightarrow \infty)$, equal to $\xi$ according to Eq. (4). The patterns of $-\varphi / \xi$ for different values of $\Xi$ are plotted in figure 3 . Surface heat transfer is assumed to be negligible for sake of simplicity.

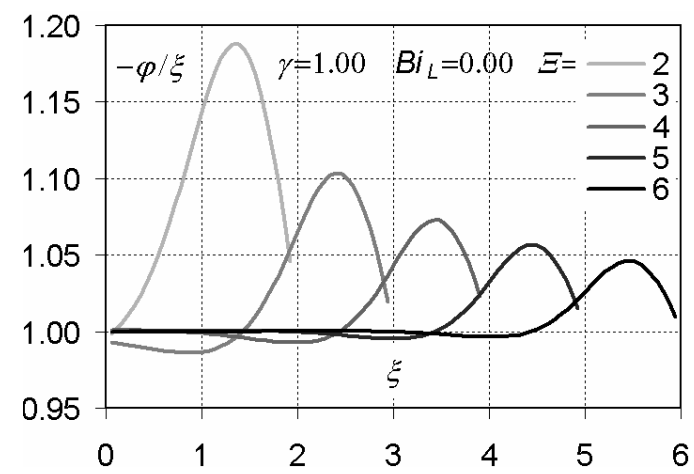

Fig. 3. Impact of the specimen length on reflection effects.

In the region close to the source $-\varphi / \xi$ is about 1 , that is reflection effects are negligible. The region widens with increasing $\Xi$. As thermal waves are exponentially damped from the source and the signal to noise ratio of temperature measurements is expected to fall below acceptable values after a few times $L, \Xi$ should not exceed $2 \div 3$ to obtain measurable reflection effects. follows:

The definition of the Biot number at the end surface opposite to the source, $B i_{L}$, can be reformulated as

$$
B i_{L}=\frac{h^{\prime} L}{\lambda} \equiv h \sqrt{\frac{1}{\lambda \rho c} \frac{t_{0}}{\pi}}
$$

$B i_{L}$ depends on $h, t_{0}$ and the so-called thermal effusivity of the material, given by the product $\lambda \rho c$ $\left[\mathrm{kg}^{2} /\left(\mathrm{K}^{2} \mathrm{~s}^{5}\right)\right]$. With natural convection, the heat transfer coefficient $h^{\prime}$ is generally below $10 \mathrm{~W} /\left(\mathrm{m}^{2} \mathrm{~K}\right)$ for a metal surface, especially if that surface is not coated to increase the emissivity. Moreover, the smallest values of the effusivity, producing the greatest values of $B i_{L}$, are about $10^{8} \mathrm{~kg}^{2} /\left(\mathrm{K}^{2} \mathrm{~s}^{5}\right)$ for metals and $10^{6} \div 10^{7} \mathrm{~kg}^{2} /\left(\mathrm{K}^{2} \mathrm{~s}^{5}\right)$ for ceramic oxides or other non-metallic material that can be tested by the Angstroem's method [18]. Using a thermoelectric source also imposes a practical upper bound to the time-cycle, equal to about one hour with metals and $5 \div 10$ min with less conductive materials, in order to limit either test duration or the impact of heat exchange with the test ambient. In the end, $B i_{L}$ is always below 0.1 in diffusivity measurements, with typical values below $0.01 \div 0.02$, and it is expected to have a secondary impact on the phase pattern (figure 4).

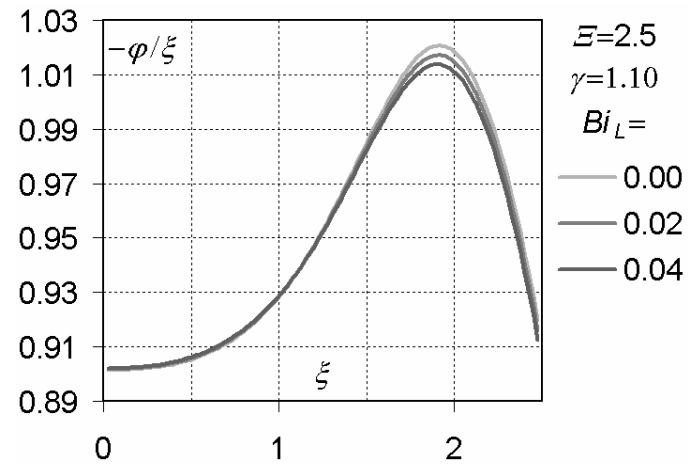

Fig. 4. Dependence of reflection effects on surface heat transfer at the end surface.

Neglecting heat loss (but not wave reflection) at the end surface opposite to the thermal source, i.e. assuming $B i_{L}=0$, the patterns of amplitude and phase along the specimen are described by the following simplified relationships: 


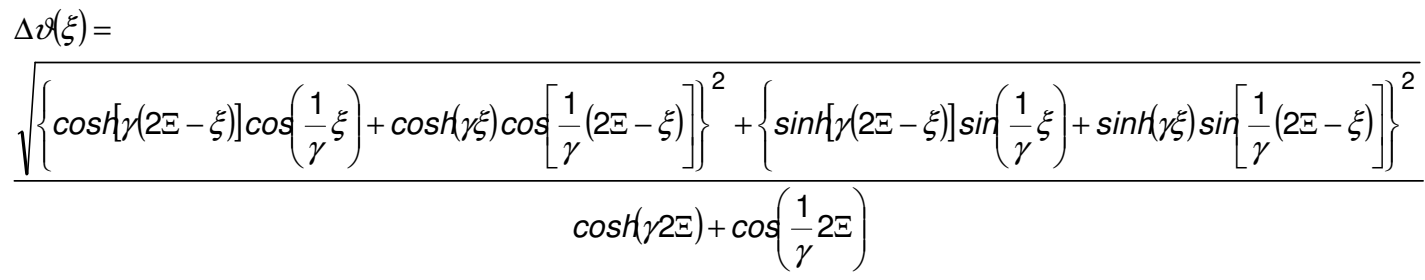

$$
\varphi(\xi)=-\arctan \left\{\frac{\sinh [\gamma(2 \Xi-\xi)] \sin \left(\frac{1}{\gamma} \xi\right)+\sinh (\gamma \xi) \sin \left[\frac{1}{\gamma}(2 \Xi-\xi)\right]}{\cosh [\gamma(2 \Xi-\xi)] \cos \left(\frac{1}{\gamma} \xi\right)+\cosh (\gamma \xi) \cos \left[\frac{1}{\gamma}(2 \Xi-\xi)\right]}\right\}
$$

Equations (43)-(44) still take into account heat exchange with the test ambient at the side surfaces through the parameter $\gamma$.

\section{Procedure to estimate the thermal diffusivity}

The thermal diffusivity is evaluated by fitting the time evolution patterns of temperature along the specimen. More specifically, amplitude and phase of the harmonic cycle are preliminarily evaluated for each position. The amplitude is then normalised by $\Delta T_{0}$ to obtain the dimensionless pattern $\Delta \vartheta(\xi)$ to be fitted with Eq. (38) or Eq. (40), whereas the phase is shifted by $\varphi_{0}$ to obtain the pattern $\varphi(\xi)$ to be fitted with Eq. (39) or Eq. (41). On the purpose, the thermal diffusion length $L$ is made explicit in the abovementioned relationships by substituting $\xi$ and $\Xi$ with $x / L$ and $X / L$, respectively. Dimensionless amplitude and phase are eventually fitted to recover $L$ and, through the definition in Eq. (5), the diffusivity value $\alpha$.

The formulation of Eq. (38)-(39) or Eq. (40)-(41) allows one to use each relationship separately in the fitting procedure, yielding two alternative and independent estimates of all unknown parameters. This permits either a cross check of the results or the fitting of one equation alone, for instance the one describing the phase patterns. In fact, phase fitting is expected to produce better results than amplitude fitting as phase measurements by infrared thermography are less affected by non-homogeneous emissivity of the specimen surface, especially when surface coating is not allowed.

Another advantage of the thermally short specimen approach is that, with non negligible heat exchange with the test ambient, the parameters $L, \gamma$ and $B i_{L}$ are not grouped in the relationships to be fitted but they can be estimated separately. This is not the case with a semi-infinite specimen, where the fitting procedure can return only the groups $\alpha_{\Delta}=\alpha / \gamma^{2}$ and $\alpha_{\varphi}=\alpha \cdot \gamma^{2}$.

The estimate procedure was validated theoretically with temperature data generated by numerical simulation. More specifically, experiments of diffusivity measurement were modelled in the Matlab programming environment through a one-dimensional finite volume approach and Cranck-Nicolson discretization of the time derivatives. Heat exchange with the test environment was considered. It was thus possible to predict the thermal transient as a result of the cycle imposed at one end of the specimen, evaluating the temperature pattern along the specimen itself at each time step. The sequences of calculated temperature patterns were eventually fitted according to the procedure described above, using a non-linear least square method again implemented in Matlab. Temperature data sets as large as permitted by current thermal cameras were generated, with frequency up to $10 \div 30 \mathrm{~Hz}$ and several hundreds of pixels along the monitored specimens surface. Values of $\Delta T_{0}$ up to $10 \div 15 \mathrm{~K}$ were used in the analyses, compatible with easy to build thermoelectric sources. A broad range of thermal diffusivity values was also considered, representative of most types of metals, from steel alloys to copper, aluminium and pure silver, and many high-conductivity non-metallic materials.

Excellent results were generally obtained with fitting of Eqs (38)-(41), achieving an accurate estimate of the diffusivity even when artificial noise was added to the numerically generated data, with gaussian distribution and average intensity comparable to that of thermal cameras with uncooled sensor $(0.1 \mathrm{~K})$. Fitting the data with Eq. (44), i.e. assuming the end surface adiabatic, also gave a discrepancy of the diffusivity estimates generally within a few percent from the actual value.

Finally, the analyses stressed out that, with non-negligible surface heat transfer, the simultaneous estimate of $L, B i_{L}$ and $\gamma$ is still possible, but the experimental conditions must be carefully chosen. The dimensionless length of the specimen, $\Xi$, must range between 1.5 and 3 . Moreover, for the success of the fitting procedure, the selection of very narrow search intervals for $B i_{L}$ and $\gamma$ is crucial. All that seems to give significant limitations, but it was verified that an estimate of $L$ and, consequently, $\alpha$ is usually possible, for instance by neglecting surface heat transfer as a first approximation or by approaching a condition of thermally semi-infinite specimen. From that preliminary estimate, a maximum value can be hypothesized for the upper bounds of $\gamma$ and $B i_{L}$, whereas the lower bounds are respectively equal to 1 and 0 . 


\section{Concluding remarks}

This work presents a mathematical model aimed at the estimate of the thermal diffusivity by the Angstroem's method when this is applied to 'thermally short' specimens, in which the temperature field is affected by reflection of thermal waves at the end opposite to that of application. If heat exchange with the test ambient is not negligible, the model can also take advantage of the effects of reflection to recover an estimate of the diffusivity from either the phase shift alone, or the amplitude decay alone. This is not possible with 'thermally semi-infinite' specimens as information from phase and amplitude must be simultaneously exploited to eliminate the effects of surface heat transfer.

From the mathematical model, a procedure to estimate the diffusivity was developed and tested theoretically by numerical simulation. Very accurate estimates of the diffusivity were generally obtained, even when a gaussian noise was added to the temperature data, with intensity comparable to that of typical infrared camera with uncooled sensor.

In the laboratory practice, the procedure that has been developed is expected to allow the use of temperature data acquired by infrared thermography in cases such as preparation of the specimen surface is not possible, or highly conductive materials available in small samples must be tested. An experimental verification is currently underway.

\section{Acknowledgements}

The author would like to thank Prof. Giovanni S. Barozzi, Dr. Luca Tarozzi and Dr. Antonio Libbra of the University of Modena and Reggio Emilia for their collaboration.

\section{REFERENCES}

[1] A.J. Angstroem. Ann. Phys. (Leipzig), 114 (1861) 513.

[2] R.W. King. A method of measuring heat conductivity, Physical Review, 6 (1915) 437-445

[3] C. Starr. An improved method for the determination of thermal diffusivities, Review of Scientific Instruments, 8 (1937) 61-64.

[4] P.H Sidles, and G.C. Danielson. Thermal diffusivity of metals at high temperature, Journal of Applied Physics, 25 (1954) 58-66.

[5] M. Cerceo, and H.M. Childers. Thermal diffusivity by electron bombardment heating, Journal of Applied Physics, 34 (1963) 1445-1449.

[6] H.R. Meddins, and J.E. Parrott. An apparatus for the high-temperature measurement of thermal diffusivity, electrical conductivity and Seebeck coefficient, Journal of Physics D: Applied Physics (British Journal of Applied Physics), 2 (1969) 691-697.

[7] J. Ebrahimi. Thermal diffusivity measurement of small silicon chips, Journal of Physics D: Applied Physics (British Journal of Applied Physics), 3 (1970) 236-239.

[8] I. Hatta, Y. Sasuga, R. Kato, and A. Maesono. Thermal diffusivity measurement of thin films by means of an ac calorimetric method, Review of Scientific Instruments, 56 (1985) 1643-1647.

[9] T.H. Johansen. Computerized analysis of thermal correlations using Peltier ac-heating, Journal of Applied Physics, 60 (1986) 2754-2761.

[10] A. Santucci, L. Verdini, and P.G. Verdini. Data-acquisition system for measurement of thermal diffusivity and propagation properties of thermal waves by a non-steady-state method, Review of Scientific Instruments, 57 (1986) 1627-1632.

[11] J.M. Belling, and L. Unsworth. Modified Angstroem's method for measurement of thermal diffusivity of materials with low conductivity, Review of Scientific Instruments, 58 (1987) 997-1002.

[12] W. Csarnetski, M. Wandelt, and W. Roetzel. Thermal wave analysis for measurement of thermal diffusivity, Proc. IEEE Instrumentation and Measurement Technology Conference, Brussel, Belgium (1996) 1195-1199.

[13] P.G. Bison, A. Muscio, and E. Grinzato. Thermal parameters estimation by heating and cooling and thermographic measurement, Proc. Thermosense XXI Conference, Orlando, U.S.A., SPIE 3700 (1999) 402408.

[14] A. Muscio, and E. Grinzato. The lock-in heating-cooling method for the measurement of the thermal diffusivity of solid materials, Heat Transfer Engineering, 23 (2002) 44-52.

[15] P.G. Bison, S. Marinetti, A. Mazzoldi, E. Grinzato, and C. Bressan. Cross-comparison of thermal diffusivity measurements by thermal methods, Infrared Physics \& Technology, 43 (2002) 127-132.

[16] A. Muscio, P.G. Bison, S. Marinetti, and E. Grinzato. Thermal diffusivity measurement in slabs using harmonic and one-dimensional propagation of thermal waves, Int. J. of Thermal Sciences, 43 (2004) 453463.

[17] H.S. Carslaw, and J.C. Jaeger. Conduction of heat in solids. Oxford University Press (1959).

[18] F.P. Incropera, and D.P. DeWitt, Fundamentals of heat and mass transfer, 4th ed., John Wiley and Sons (1996). 
http://dx.doi.org/10.21611/qirt.2008.15_09_12 